Xiaoming Wang and Zhi-Qiang Wang*

\title{
Normalized multi-bump solutions for saturable Schrödinger equations
}

https://doi.org/10.1515/anona-2020-0054

Received December 21, 2018; accepted October 28, 2019.

Abstract: In this paper, we are concerned with the existence of multi-bump solutions for a class of semiclassical saturable Schrödinger equations with an density function:

$$
-\Delta v+\Gamma \frac{I(\varepsilon x)+v^{2}}{1+I(\varepsilon x)+v^{2}} v=\lambda v, x \in \mathbb{R}^{2} .
$$

We prove that, with the density function being radially symmetric, for given integer $k \geq 2$ there exist a family of non-radial, $k$-bump type normalized solutions (i.e., with the $L^{2}$ constraint) which concentrate at the global maximum points of density functions when $\varepsilon \rightarrow 0^{+}$. The proof is based on a variational method in particular on a convexity technique and the concentration-compactness method.

Keywords: Schrödinger equations; saturable nonlinearity; semiclassical states; multi-bump solution; concentrations

MSC: $35 J 20,35060$

\section{Introduction and main results}

This paper deals with the existence of solutions $(v, \lambda) \in H^{1}\left(\mathbb{R}^{2}, \mathbb{R}\right) \times \mathbb{R}$ to the following nonlinear eigenvalue problem with saturable nonlinearity

$$
-\Delta v+\Gamma \frac{I(\varepsilon x)+v^{2}}{1+I(\varepsilon x)+v^{2}} v=\lambda v, x \in \mathbb{R}^{2},
$$

where $\varepsilon$ is a small parameter (related to the Planck constant), $\Gamma$ is a coupling constant, and $I(x)$, the density function, is a bounded continuous function. This model describes paraxial counter-propagating beams in isotropic local media (e.g., [1-5]). An interesting issue concerning (1.1) is the existence of semiclassical states, which concerns the study of (1.1) for small $\varepsilon>0$. From the physics point of view, semiclassical states describe a sort of transition from quantum mechanics to classical mechanics as the parameter $\varepsilon$ goes to zero. In (1.1), one can either consider the parameter $\lambda \in \mathbb{R}$ to be given, or to be an unknown of the problem. In this paper, we study the latter case, i.e., we look for normalized solutions with the $L^{2}$ norm prescribed and $\lambda$ as a Lagrange multiplier. For small $\varepsilon>0$ in (1.1), we will make a first attempt to study the existence and concentration behavior of multi-bump type solutions in $H^{1}\left(\mathbb{R}^{2}\right)$. We refer [6-8] for results on the problems of saturable nonlinearity without constraints and references therein such as existence and concentration property of solutions.

\footnotetext{
Xiaoming Wang, School of Mathematics \& Computer Science, Shangrao Normal University, Shangrao 334001, China, E-mail: wxmsuda03@163.com

*Corresponding Author: Zhi-Qiang Wang, College of Mathematics and Informatics, Fujian Normal University, Fuzhou 350117, China, and Department of Mathematics and Statistics, Utah State University, Logan, UT 84322, USA, E-mail:

zhi-qiang.wang@usu.edu
} 
The main goal of this paper is to establish the existence and concentration behavior of multi-bump solutions with a localizing potential $I(\varepsilon x)$ for small $\varepsilon>0$

$$
-\Delta u+\Gamma \frac{I(\varepsilon x)+u^{2}}{1+I(\varepsilon x)+u^{2}} u=\lambda u, \quad \text { for } x \in \mathbb{R}^{2} .
$$

It is well known that equation (1.2) is the Euler-Lagrange equation of the following minimization problem subject to a $L^{2}$ constraint

$$
m_{\varepsilon}(\Gamma, I)=\inf \left\{E_{\varepsilon}(\rho) \mid \rho \in H^{1}\left(\mathbb{R}^{2}\right), \int_{\mathbb{R}^{2}} \rho^{2}=1\right\},
$$

where

$$
E_{\varepsilon}(\rho)=\int_{\mathbb{R}^{2}}|\nabla \rho|^{2}+\Gamma\left[\rho^{2}-\ln \left(1+\frac{\rho^{2}}{1+I(\varepsilon x)}\right)\right] .
$$

Also observe that in this case the parameter $\lambda \in \mathbb{R}$ depending on $\varepsilon$ (so in what follows, we denote $\lambda=\lambda_{\varepsilon}$ ), comes from problem (1.3) and can be interpreted as a Lagrange multiplier. Among all possible standing waves for equation (1.2), typically the most relevant are ground state solutions. Recently, in [9], by a global minimization method, we have obtained the existence and concentration behavior of positive normalized ground state solutions of equation (1.2) in $H^{1}\left(\mathbb{R}^{2}\right)$ for small $\varepsilon>0$, under the condition

(B1) $I(x)$ satisfies

$$
I_{2}=\max _{x \in \mathbb{R}^{2}} I(x)>\limsup _{|x| \rightarrow+\infty} I(x)=I_{\infty} \geq I_{1}=\inf _{\mathbb{R}^{2}} I(x)>-1 .
$$

We remark that in most cases the global minimizers are not necessarily multi-bump solutions, and that when $I(x)$ is radially symmetric the global minimizers may be radially symmetric functions. In this paper we investigate conditions on $I(x)=I(|x|)$ under which the minimizers are non-radial and multi-bump type solutions. In order to solve this problem, we introduce a local minimization procedure and work on a subspace of $H^{1}\left(\mathbb{R}^{2}\right)$. The main ideas come from the methods introduced in $[10,11]$ of the second author. This local minimization procedure has been successfully used to treat nonlinear Dirichlet problems $[10,12]$ and nonlinear Neumann problems [13]. The advantage of this method is that we can get qualitative properties of the solutions constructed such as the concentration behavior and the shape of solutions with a discrete number of bumps. However this type of method and results have not been studied before for normalized solutions and there are new difficulties which require new ideas and variational techniques.

Let $k \geq 2$ be a fixed positive integer. We define

$$
H_{k}^{1}\left(\mathbb{R}^{2}\right)=\left\{u \in H^{1}\left(\mathbb{R}^{2}\right) \mid u\left(g^{-1} x\right)=u(x) \text {, a.e. in } \mathbb{R}^{2}, \text { for all } g \in G_{k}\right\},
$$

where

$$
G_{k}=\left\{g \in O(2) \mid g\left(x_{1}, x_{2}\right)=\left(x_{1} \cos \frac{2 \pi l}{k}+x_{2} \sin \frac{2 \pi l}{k},-x_{1} \sin \frac{2 \pi l}{k}+x_{2} \cos \frac{2 \pi l}{k}\right),\left(x_{1}, x_{2}\right) \in \mathbb{R}^{2}\right\},
$$

$l=1,2, \ldots, k$, and $O(2)$ is the group of orthogonal transformations in $\mathbb{R}^{2}$. It is easy to see that $G_{k}$ is a cyclic group of order $k$. In order to get multi-bump type solutions, we consider the following minimization problem

$$
\begin{aligned}
m_{\Gamma, k}(\varepsilon) & =\inf _{u \in H_{k}^{1}\left(\mathbb{R}^{2}\right), \int_{\mathbb{R}^{2}} u^{2}=1} H_{\varepsilon}(u) \\
& =\inf _{u \in H_{k}^{1}\left(\mathbb{R}^{2}\right), \int_{\mathbb{R}^{2}} u^{2}=1} \int_{\mathbb{R}^{2}}|\nabla u|^{2}+\Gamma\left[u^{2}-\ln \left(1+\frac{u^{2}}{1+I(\varepsilon x)}\right)\right] d x .
\end{aligned}
$$

If (B1) is satisfied and $I(x)=I(|x|) \in C\left(\mathbb{R}^{2}, \mathbb{R}\right) \cap L^{\infty}\left(\mathbb{R}^{2}, \mathbb{R}\right)$ is radially symmetric, using a similar procedure as in the proof of Theorem 2.1 in [9], we may deduce the existence result of a minimizer for the above minimization problem $m_{\Gamma, k}(\varepsilon)$. But to show the minimizers are non-radial and of multi-bump type we would need additional conditions on the density function $I(x)$.

By (B1) we deduce that the maximum value of $I(x)$ must be obtained on a bounded closed set. We suppose that 
(B2) $I(x)=I(|x|)$ is radial and achieves its unique maximum on $S^{1}=\left\{x \in \mathbb{R}^{2}|| x \mid=1\right\}$, and there exist $\delta_{0}>0$ and $r_{0}>0$ such that $I(x) \geq I_{\infty}+\delta_{0}$ for ||$x|-1| \leq r_{0}$.

Then we have the following Theorem.

Theorem 1.1. Assume that $I(x)$ satisfies (B1)-(B2). For any integer $k \geq 2$ fixed, there exists $\Gamma_{0}=\Gamma_{0}\left(I_{\infty}, k, \delta_{0}, r_{0}\right)$ (but independent of $\varepsilon>0, I_{1}$ and $I(0)$ ), for each fixed $\Gamma<\Gamma_{0}$, there exist $\varepsilon_{0}=\varepsilon_{0}(\Gamma)>0$ and $\alpha_{0}=\alpha_{0}(\Gamma)>0$ such that if $0<\varepsilon<\varepsilon_{0}$ and $0<1+I(0)<\alpha_{0}, m_{\Gamma, k}(\varepsilon)$ has a minimizer solution $u_{\varepsilon} \in H_{k}^{1}\left(\mathbb{R}^{2}\right)$ satisfying

(i)

$$
\lim _{\varepsilon \rightarrow 0^{+}} m_{\Gamma, k}(\varepsilon)=k m\left(\frac{1}{k}, I_{2}\right),
$$

where

$$
m\left(\frac{1}{k}, I_{2}\right)=\inf _{u \in H^{1}\left(\mathbb{R}^{2}\right), \int_{\mathbb{R}^{2}} u^{2}=\frac{1}{k}} \int_{\mathbb{R}^{2}}|\nabla u|^{2}+\Gamma\left[u^{2}-\ln \left(1+\frac{u^{2}}{1+I_{2}}\right)\right] d x .
$$

(ii) $u_{\varepsilon}$ is of $k$-bump type in the sense that $u_{\varepsilon}$ has exactly $k$ maximum points which form a $G_{k}$-orbit $G_{k}\left(y_{\varepsilon}\right)$ for some $y_{\varepsilon} \in \mathbb{R}^{2}$ satisfying $\left|\varepsilon y_{\varepsilon}\right| \rightarrow 1$ and up to subsequences

$$
\left\|u_{\varepsilon}-\sum_{i=1}^{k} w\left(x+\frac{g_{i} e_{1}}{\varepsilon}\right)\right\|_{H^{1}\left(\mathbb{R}^{2}\right)} \rightarrow 0 \text {, as } \varepsilon \rightarrow 0^{+},
$$

where $w(x)$ is a minimizer of $m\left(\frac{1}{k}, I_{2}\right)$ and $e_{1} \in S^{1}=\left\{x \in \mathbb{R}^{2}|| x \mid=1\right\}$.

The existence of a minimizer follows from the work of [14] (will be stated in Theorem 2.1), and we mainly concern whether there are multi-bumps for the local minimizers $u_{\varepsilon} \in H_{k}^{1}\left(\mathbb{R}^{2}\right)$ as $\varepsilon \in\left(0, \varepsilon_{0}\right)$. Generally speaking, this conclusion is not necessarily true. For example, if only (B1) is satisfied, by Theorem $2.4 \mathrm{in}$ [14], we see that $u_{\varepsilon} \in H_{k}^{1}\left(\mathbb{R}^{2}\right)$ may be a radially symmetric solution and has only one bump centered at the origin. Therefore, in order to construct multi-bump solutions, we need to impose some additional conditions on $I(x)$. We prove that (B2) and the condition on $I(0)$ are sufficient to assure the minimizers are of multi-bump type solutions.

This paper is structured as follows. In Section 2 we will present and show some useful lemmas which are useful for the proof of Theorem 1.1. Afterwards, in Section 3 we will give the proof of Theorem 1.1.

Notation. Throughout this paper, we denote by $C$ a positive constant, which may vary from line to line; all integrals are taken over $\mathbb{R}^{2}$; All $d x$ in the integrals are omitted; $L^{p} \equiv L^{p}\left(\mathbb{R}^{2}\right)(1 \leq p<+\infty)$ is the usual Lebesgue space with the norm $\|u\|_{p}^{p}=\int_{\mathbb{R}^{2}}|u|^{p} ; H^{1} \equiv H^{1}\left(\mathbb{R}^{2}\right)$ denotes the uaual Sobolev space with the norm $\|u\|^{2}=\int_{\mathbb{R}^{2}}\left(|\nabla u|^{2}+|u|^{2}\right) ; o_{n}(1)\left(\right.$ resp. $\left.o_{\varepsilon}(1)\right)$ will denote a generic infinitesimal as $n \rightarrow \infty\left(\right.$ resp. $\left.\varepsilon \rightarrow 0^{+}\right) ; \rightarrow$ denotes the strong convergence and $\rightarrow$ the weak convergence.

\section{Some technical results}

In this section, we will establish several lemmas, which will be useful to prove Theorem 1.1 in next Section. First using a similar procedure as in the proof of Theorem 2.1 in [9], we may deduce the existence result of a minimizer for $m_{\Gamma, k}(\varepsilon)$.

Theorem 2.1. Suppose that $I(x)=I(|x|)$ satisfies (B1)-(B2). Then for given integer $k \geq 2$, there exists $\Gamma_{0}^{\prime}=$ $\Gamma_{0}^{\prime}\left(I_{\infty}, k\right)<0$ (independent of $\varepsilon>0, I_{1}$ and $I(0)$ ), for each fixed $\Gamma<\Gamma_{0}^{\prime}$, there exists $\varepsilon_{0}=\varepsilon_{0}(\Gamma)>0$ such that for all $\varepsilon \in\left(0, \varepsilon_{0}\right)$, the minimization problem

$$
\begin{aligned}
m_{\Gamma, k}(\varepsilon) & =\inf _{u \in H_{k}^{1}\left(\mathbb{R}^{2}\right), \int_{\mathbb{R}^{2}} u^{2}=1} H_{\varepsilon}(u) \\
& =\inf _{u \in H_{k}^{1}\left(\mathbb{R}^{2}\right), \int_{\mathbb{R}^{2}} u^{2}=1} \int_{\mathbb{R}^{2}}|\nabla u|^{2}+\Gamma\left[u^{2}-\ln \left(1+\frac{u^{2}}{1+I(\varepsilon x)}\right)\right]
\end{aligned}
$$


possesses a solution $u_{\varepsilon}$, which solves equation (1.2) for some $\lambda<0$.

This follows from the proofs in [9], in which $I(x)$ is fixed throughout the proof there. As we need to place a condition on $I(0)$ as in Theorem 1.1, closer examination tells us that the proof of the above result works through if we fix the property of $I$ in the neighborhood of the maximum points while allowing changes of $I(0)$. We omit the details here.

Next in order to analyze the asymptotic behavior of the minimizers $u_{\varepsilon}$ we prepare some estimates.

Lemma 2.1. For given integer $k \geq 2$, there exist $\Gamma_{1}=\Gamma_{1}\left(I_{2}, k\right)<0$ and $0<a=a\left(I_{2}, k\right)<\frac{1}{k}$, such that for each fixed $\Gamma<\Gamma_{1}$,

$$
m\left(\frac{1}{k}, I_{2}\right)=\inf _{u \in H^{1}\left(\mathbb{R}^{2}\right), \int_{\mathbb{R}^{2}} u^{2}=\frac{1}{k}} \int_{\mathbb{R}^{2}}|\nabla u|^{2}+\Gamma\left[u^{2}-\ln \left(1+\frac{u^{2}}{1+I_{2}}\right)\right]
$$

is achieved by $u_{k}$ which is radially symmetric. Moreover,

$$
k m\left(\frac{1}{k}, I_{2}\right)<\frac{\Gamma I_{2}}{1+I_{2}} .
$$

In particular, we have

$$
m\left(\frac{1}{k}, I_{2}\right) \leq \frac{a \Gamma}{2} \text { and } \frac{k a \Gamma}{2}+1<0 .
$$

Proof. Using the same arguments as Theorem 2.1 in [14], we know that there exists $\Gamma_{1}^{\prime}=\Gamma_{1}^{\prime}\left(I_{2}, k\right)<0$, such that for each fixed $\Gamma<\Gamma_{1}^{\prime}$ the minimization problem $m\left(\frac{1}{k}, I_{2}\right)$ is attained by $u_{k}$. In addition, by Theorem 2.3 in [14], $u_{k}$ is a radially symmetric function.

Moreover, we have

$$
\begin{aligned}
m\left(\frac{1}{k}, I_{2}\right) & =\inf _{u \in H^{1}\left(\mathbb{R}^{2}\right), \int_{\mathbb{R}^{2}} u^{2}=\frac{1}{k}} \int_{\mathbb{R}^{2}}|\nabla u|^{2}+\Gamma\left[u^{2}-\ln \left(1+\frac{u^{2}}{1+I_{2}}\right)\right] \\
& =\inf _{u \in H^{1}\left(\mathbb{R}^{2}\right), \int_{\mathbb{R}^{2}} u^{2}=\frac{1}{k}} \int_{\mathbb{R}^{2}}|\nabla u|^{2}+\Gamma\left[\frac{u^{2}}{1+I_{2}}-\ln \left(1+\frac{u^{2}}{1+I_{2}}\right)\right]+\int_{\mathbb{R}^{2}} \frac{\Gamma I_{2} u^{2}}{1+I_{2}} \\
& <\frac{\Gamma I_{2}}{k\left(1+I_{2}\right)},
\end{aligned}
$$

and this implies

$$
k m\left(\frac{1}{k}, I_{2}\right)<\frac{\Gamma I_{2}}{1+I_{2}} .
$$

On the other hand, we may find a sequence of functions $u_{n}$ such that $\left\|u_{n}\right\|_{2}^{2}=\frac{1}{k}$ and $a_{n}=\int_{\mathbb{R}^{2}}\left[u_{n}^{2}-\ln (1+\right.$ $\left.\left.\frac{u_{n}^{2}}{1+I}\right)\right] \rightarrow \frac{1}{k}$ as $n \rightarrow \infty$. In fact, this can be done by choosing $v(x) \in C_{0}^{\infty}\left(B_{1}(0)\right)$ such that $\int_{\mathbb{R}^{2}} v^{2}=\frac{1}{k}$, and setting $u_{n}(x)=n v(n x)$, then

$$
\begin{aligned}
a_{n} & =\int_{\mathbb{R}^{2}}\left[u_{n}^{2}-\ln \left(1+\frac{u_{n}^{2}}{1+I_{2}}\right)\right]=\int_{\mathbb{R}^{2}}\left[n^{2} v^{2}(n x)-\ln \left(1+\frac{n^{2} v^{2}(n x)}{1+I_{2}}\right)\right] \\
& =\int_{\mathbb{R}^{2}}\left[v^{2}(y)-\frac{1}{n^{2}} \ln \left(1+\frac{n^{2} v^{2}(y)}{1+I_{2}}\right)\right]=\frac{1}{k}-\int_{\mathbb{R}^{2}} \frac{1}{n^{2}} \ln \left(1+\frac{n^{2} v^{2}(y)}{1+I_{2}}\right) \\
& \rightarrow \frac{1}{k}, \text { as } n \rightarrow \infty .
\end{aligned}
$$

Here we used the fact for $b>0$ fixed,

$$
\frac{\ln (1+b \rho)}{\rho} \rightarrow 0, \text { as } \rho \rightarrow+\infty \text {. }
$$

Hence, there is a function $u_{0}$ with $\left\|u_{0}\right\|_{2}^{2}=\frac{1}{k}$ and

$$
0<a \equiv a\left(I_{2}, k\right)=\int_{\mathbb{R}^{2}}\left[u_{0}^{2}-\ln \left(1+\frac{u_{0}^{2}}{1+I_{2}}\right)\right]<\frac{1}{k} .
$$


Noticing that

$$
\lim _{\Gamma \rightarrow-\infty} \frac{m\left(\frac{1}{k}, I_{2}\right)}{\Gamma} \geq \lim _{\Gamma \rightarrow-\infty} \frac{\int_{\mathbb{R}^{2}}\left|\nabla u_{0}\right|^{2}+\Gamma\left[u_{0}^{2}-\ln \left(1+\frac{u_{0}^{2}}{1+I_{2}}\right)\right]}{\Gamma}=a>0 .
$$

Therefore, there exists $\Gamma_{1}^{\prime \prime}=\Gamma_{1}^{\prime \prime}\left(I_{2}, k\right)$, such that

$$
m\left(\frac{1}{k}, I_{2}\right) \leq \frac{a \Gamma}{2} \text { and } \frac{k a \Gamma}{2}+1<0
$$

if $\Gamma<\Gamma_{1}^{\prime \prime}$.

As a result, taking

$$
\Gamma_{1}=\Gamma_{1}\left(I_{2}, k\right) \equiv \min \left\{\Gamma_{1}^{\prime}\left(I_{2}, k\right), \Gamma_{1}^{\prime \prime}\left(I_{2}, k\right)\right\},
$$

we can get the desired results for Lemma 2.1.

Now in the following, for given $k \in \mathbb{N}^{+}$, we always fix

$$
\Gamma<\Gamma_{0}=\Gamma_{0}\left(I_{2}, I_{\infty}, k\right) \equiv \min \left\{\Gamma_{1}\left(I_{2}, k\right), \Gamma_{0}^{\prime}\left(I_{\infty}, k\right)\right\}<0,
$$

where $\Gamma_{0}^{\prime}\left(I_{\infty}, k\right)$ is given in Theorem 2.1.

We remark that by Theorem 2.1 and (B2), we know that no matter how $I(x)$ changes outside the neighborhood of ||$x|-1| \leq 2 r_{0}$, for each fixed $\Gamma<\Gamma_{0}$, there exists $\varepsilon_{0}=\varepsilon_{0}(\Gamma)>0$, such that $m_{\Gamma, k}(\varepsilon)$ is always achieved by some $u_{\varepsilon} \in H_{k}^{1}\left(\mathbb{R}^{2}\right)$ for $\varepsilon \in\left(0, \varepsilon_{0}\right)$. At the same time, by Theorem 2.1, we know that $u_{\varepsilon}$ satisfies

$$
-\triangle u_{\varepsilon}+\Gamma \frac{I(\varepsilon x)+u_{\varepsilon}^{2}}{1+I(\varepsilon x)+u_{\varepsilon}^{2}} u_{\varepsilon}=\lambda_{\varepsilon} u_{\varepsilon}, x \in \mathbb{R}^{2},
$$

where $\lambda_{\varepsilon}$ is associated Lagrange multiplier.

Next, we start to study the qualitative properties for the minimizer $u_{\varepsilon}$ of $m_{\Gamma, k}(\varepsilon)$.

Lemma 2.2. It holds that

$$
\varlimsup_{\varepsilon \rightarrow 0^{+}} m_{\Gamma, k}(\varepsilon) \leq k m\left(\frac{1}{k}, I_{2}\right) .
$$

Proof. Taking $e_{1} \in S^{1}$ and the $G_{k}$-orbit of $e_{1}$ containing exactly $k$ points, $\left\{g_{i} e_{1} \mid i=1,2, \ldots, k\right\}$, and defining

$$
U_{\varepsilon}(x)=\sum_{i=1}^{k} w\left(x+\frac{g_{i} e_{1}}{\varepsilon}\right),
$$

where $w(x) \in H^{1}\left(\mathbb{R}^{2}\right)$ is the minimizer of $m\left(\frac{1}{k}, I_{2}\right)$ so $w(x) \rightarrow 0$ as $|x| \rightarrow \infty$. Moreover, by Lemma 2.1, $w$ is radially symmetric. Since

$$
\lim _{\varepsilon \rightarrow 0^{+}}\left|\frac{g_{i} e_{1}-g_{j} e_{1}}{\varepsilon}\right|=+\infty \text { for } i \neq j
$$

we have

$$
\begin{aligned}
\left\|U_{\varepsilon}(x)\right\|_{2}^{2} & =\int_{\mathbb{R}^{2}}\left|\sum_{i=1}^{k} w\left(x+\frac{g_{i} e_{1}}{\varepsilon}\right)\right|^{2} d x \\
& =\sum_{i=1}^{k} \int_{\mathbb{R}^{2}}\left|w\left(x+\frac{g_{i} e_{1}}{\varepsilon}\right)\right|^{2} d x+o_{\varepsilon}(1) \\
& \rightarrow 1, \text { as } \varepsilon \rightarrow 0^{+} .
\end{aligned}
$$

Setting

$$
V_{\varepsilon}(x)=\frac{U_{\varepsilon}(x)}{\left\|U_{\varepsilon}(x)\right\|_{2}},
$$

then $\left\|V_{\varepsilon}(x)\right\|_{2}^{2}=1$ and $V_{\varepsilon}(x) \in H_{k}^{1}\left(\mathbb{R}^{2}\right)$. 
Therefore, according to $I(x)=I(|x|)$ satisfying (B2), we have

$$
\begin{aligned}
& m_{\Gamma, k}(\varepsilon) \leq H_{\varepsilon}\left(V_{\varepsilon}\right)=\int_{\mathbb{R}^{2}}\left|\nabla V_{\varepsilon}\right|^{2}+\Gamma\left[V_{\varepsilon}^{2}-\ln \left(1+\frac{V_{\varepsilon}^{2}}{1+I(\varepsilon x)}\right)\right] \\
& =\int_{\mathbb{R}^{2}}\left|\nabla \frac{U_{\varepsilon}}{\left\|U_{\varepsilon}(x)\right\|_{2}}\right|^{2}+\Gamma\left[\frac{U_{\varepsilon}^{2}}{\left\|U_{\varepsilon}(x)\right\|_{2}^{2}}-\ln \left(1+\frac{U_{\varepsilon}^{2}}{\left\|U_{\varepsilon}(x)\right\|_{2}^{2}(1+I(\varepsilon x))}\right)\right] \\
& =\int_{\mathbb{R}^{2}} \frac{\left|\sum_{i=1}^{k} \nabla w\left(x+\frac{g_{i} e_{1}}{\varepsilon}\right)\right|^{2}}{\left\|U_{\varepsilon}(x)\right\|_{2}^{2}}+\Gamma\left[\frac{\left|\sum_{i=1}^{k} w\left(x+\frac{g_{i} e_{1}}{\varepsilon}\right)\right|^{2}}{\left\|U_{\varepsilon}(x)\right\|_{2}^{2}}-\ln \left(1+\frac{\left|\sum_{i=1}^{k} w\left(x+\frac{g_{i} e_{1}}{\varepsilon}\right)\right|^{2}}{\left\|U_{\varepsilon}(x)\right\|_{2}^{2}(1+I(\varepsilon x))}\right)\right] \\
& =\sum_{i=1}^{k} \int_{\mathbb{R}^{2}} \frac{\left|\nabla w\left(x+\frac{g_{i} e_{1}}{\varepsilon}\right)\right|^{2}}{\left\|U_{\varepsilon}(x)\right\|_{2}^{2}}+\Gamma\left[\frac{\left|w\left(x+\frac{g_{i} e_{1}}{\varepsilon}\right)\right|^{2}}{\left\|U_{\varepsilon}(x)\right\|_{2}^{2}}-\ln \left(1+\frac{\left|w\left(x+\frac{g_{i} e_{1}}{\varepsilon}\right)\right|^{2}}{\left.\left\|U_{\varepsilon}(x)\right\|\right|_{2} ^{2}(1+I(\varepsilon x))}\right)\right]+o_{\varepsilon}(1) \\
& =\sum_{i=1}^{k} \int_{\mathbb{R}^{2}} \frac{|\nabla w(x)|^{2}}{\left\|U_{\varepsilon}(x)\right\|_{2}^{2}}+\Gamma\left[\frac{|w(x)|^{2}}{\left\|U_{\varepsilon}(x)\right\|_{2}^{2}}-\ln \left(1+\frac{|w(x)|^{2}}{\left\|U_{\varepsilon}(x)\right\|_{2}^{2}\left(1+I\left(\varepsilon x-g_{i} e_{1}\right)\right)}\right)\right]+o_{\varepsilon}(1) \\
& \rightarrow \sum_{i=1}^{k} \int_{\mathbb{R}^{2}}|\nabla w|^{2}+\Gamma\left[w^{2}-\ln \left(1+\frac{w^{2}}{1+I\left(g_{i} e_{1}\right)}\right)\right]\left(\text { as } \varepsilon \rightarrow 0^{+}\right) \\
& =k \int_{\mathbb{R}^{2}}|\nabla w|^{2}+\Gamma\left[w^{2}-\ln \left(1+\frac{w^{2}}{1+I_{2}}\right)\right]=k m\left(\frac{1}{k}, I_{2}\right) .
\end{aligned}
$$

Thus,

$$
\varlimsup_{\varepsilon \rightarrow 0} m_{\Gamma, k}(\varepsilon) \leq k m\left(\frac{1}{k}, I_{2}\right) .
$$

Lemma 2.3. There exists $0<\varepsilon_{1} \leq \varepsilon_{0}$, such that for $\varepsilon \in\left(0, \varepsilon_{1}\right)$, the Lagrange multiplier $\lambda_{\varepsilon}$ in (2.3) satisfies

$$
m\left(1, I_{2}\right)+\frac{\Gamma}{1+I_{1}} \leq \lambda_{\varepsilon} \leq \frac{k a \Gamma}{2}+1<0,
$$

where $a>0$ is given in Lemma 2.1.

Proof. By Lemma 2.2, we know that there exists $0<\varepsilon_{1} \leq \varepsilon_{0}$, such that for $\varepsilon \in\left(0, \varepsilon_{1}\right)$, we have

$$
m_{\Gamma, k}(\varepsilon) \leq k m\left(\frac{1}{k}, I_{2}\right)+1 .
$$

Therefore, by (2.2), we have

$$
\begin{aligned}
\lambda_{\varepsilon} & =\lambda_{\varepsilon} \int_{\mathbb{R}^{2}} u_{\varepsilon}^{2}=\int_{\mathbb{R}^{2}}\left|\nabla u_{\varepsilon}\right|^{2}+\Gamma \frac{I(\varepsilon x)+u_{\varepsilon}^{2}}{1+I(\varepsilon x)+u_{\varepsilon}^{2}} u_{\varepsilon}^{2} \\
& =\int_{\mathbb{R}^{2}}\left|\nabla u_{\varepsilon}^{2}\right|+\Gamma\left[u_{\varepsilon}^{2}-\ln \left(1+\frac{u_{\varepsilon}^{2}}{1+I(\varepsilon x)}\right)\right]+\Gamma \int_{\mathbb{R}^{2}}\left[\frac{I(\varepsilon x)+u_{\varepsilon}^{2}}{1+I(\varepsilon x)+u_{\varepsilon}^{2}} u_{\varepsilon}^{2}-u_{\varepsilon}^{2}+\ln \left(1+\frac{u_{\varepsilon}^{2}}{1+I(\varepsilon x)}\right)\right] \\
& =m_{\Gamma, k}(\varepsilon)+\Gamma \int_{\mathbb{R}^{2}}\left[\frac{I(\varepsilon x)+u_{\varepsilon}^{2}}{1+I(\varepsilon x)+u_{\varepsilon}^{2}} u_{\varepsilon}^{2}-u_{\varepsilon}^{2}+\ln \left(1+\frac{u_{\varepsilon}^{2}}{1+I(\varepsilon x)}\right)\right] \\
& =m_{\Gamma, k}(\varepsilon)+\Gamma \int_{\mathbb{R}^{2}}\left[\ln \left(1+\frac{u_{\varepsilon}^{2}}{1+I(\varepsilon x)}\right)-\frac{u_{\varepsilon}^{2}}{1+I(\varepsilon x)+u_{\varepsilon}^{2}}\right] \\
& \leq m_{\Gamma, k}(\varepsilon) \leq k m\left(\frac{1}{k}, I_{2}\right)+1 \\
& \leq \frac{k a \Gamma}{2}+1<0 .
\end{aligned}
$$


Here we have used $\int_{\mathbb{R}^{2}} u_{\varepsilon}^{2}=1$ and $\int_{\mathbb{R}^{2}}\left[\ln \left(1+\frac{u_{\varepsilon}^{2}}{1+I(\varepsilon x)}\right)-\frac{u_{\varepsilon}^{2}}{1+I(\varepsilon x)+u_{\varepsilon}^{2}}\right] \geq 0$.

On the other hand, according to (2.3), we obtain

$$
\begin{aligned}
\lambda_{\varepsilon} & =\lambda_{\varepsilon} \int_{\mathbb{R}^{2}} u_{\varepsilon}^{2}=\int_{\mathbb{R}^{2}}\left|\nabla u_{\varepsilon}\right|^{2}+\Gamma \frac{I(\varepsilon x)+u_{\varepsilon}^{2}}{1+I(\varepsilon x)+u_{\varepsilon}^{2}} u_{\varepsilon}^{2} \\
& =\int_{\mathbb{R}^{2}}\left|\nabla u_{\varepsilon}^{2}\right|+\Gamma\left[u_{\varepsilon}^{2}-\ln \left(1+\frac{u_{\varepsilon}^{2}}{1+I(\varepsilon x)}\right)\right]+\Gamma \int_{\mathbb{R}^{2}}\left[\frac{I(\varepsilon x)+u_{\varepsilon}^{2}}{1+I(\varepsilon x)+u_{\varepsilon}^{2}} u_{\varepsilon}^{2}-u_{\varepsilon}^{2}+\ln \left(1+\frac{u_{\varepsilon}^{2}}{1+I(\varepsilon x)}\right)\right] \\
& \geq m_{\Gamma, k}(\varepsilon)+\Gamma \int_{\mathbb{R}^{2}}\left[\frac{1+I(\varepsilon x)+u_{\varepsilon}^{2}}{1+I(\varepsilon x)+u_{\varepsilon}^{2}} u_{\varepsilon}^{2}-u_{\varepsilon}^{2}+\ln \left(1+\frac{u_{\varepsilon}^{2}}{1+I(\varepsilon x)}\right)\right] \\
& =m_{\Gamma, k}(\varepsilon)+\Gamma \int_{\mathbb{R}^{2}} \ln \left(1+\frac{u_{\varepsilon}^{2}}{1+I(\varepsilon x)}\right) \geq m_{\Gamma, k}(\varepsilon)+\Gamma \int_{\mathbb{R}^{2}} \ln \left(1+\frac{u_{\varepsilon}^{2}}{1+I_{1}}\right) \\
& \geq m_{\Gamma, k}(\varepsilon)+\Gamma \int_{\mathbb{R}^{2}} \frac{u_{\varepsilon}^{2}}{1+I_{1}} \\
& \geq m\left(1, I_{2}\right)+\frac{\Gamma}{1+I_{1}} .
\end{aligned}
$$

Therefore, we have finished the proof of Lemma 2.3.

Lemma 2.4. There exists $0<\varepsilon_{2} \leq \varepsilon_{0}$, such that for $\varepsilon \in\left(0, \varepsilon_{2}\right)$, the minimizer $u_{\varepsilon}$ of $m_{\Gamma, k}(\varepsilon)$ satisfies

$$
\left\|u_{\varepsilon}\right\|_{H_{k}^{1}\left(\mathbb{R}^{2}\right)} \leq L=\sqrt{2+\frac{|\Gamma|}{1+I_{2}}} .
$$

Proof. By Lemma 2.2, there exists $0<\varepsilon_{2} \leq \varepsilon_{0}$, such that for $\varepsilon \in\left(0, \varepsilon_{2}\right)$, we have

$$
\begin{aligned}
1+k m\left(\frac{1}{k}, I_{2}\right) & \geq m_{\Gamma, k}(\varepsilon)=\int_{\mathbb{R}^{2}}\left|\nabla u_{\varepsilon}(x)\right|^{2}+\Gamma\left[u_{\varepsilon}^{2}(x)-\ln \left(1+\frac{u_{\varepsilon}^{2}(x)}{1+I(\varepsilon x)}\right)\right] \\
& \geq \int_{\mathbb{R}^{2}}\left|\nabla u_{\varepsilon}(x)\right|^{2}+\Gamma .
\end{aligned}
$$

Here we have used the fact that $\int_{\mathbb{R}^{2}} u_{\varepsilon}^{2}(x)=1$. Thus, by Lemma 2.1, we have

$$
\begin{aligned}
\left\|u_{\varepsilon}\right\|_{H_{k}^{1}\left(\mathbb{R}^{2}\right)}^{2} & =\int_{\mathbb{R}^{2}}\left|\nabla u_{\varepsilon}(x)\right|^{2}+u_{\varepsilon}^{2}(x) \\
& \leq 2-\Gamma+k m\left(\frac{1}{k}, I_{2}\right) \\
& \leq 2-\Gamma+\frac{\Gamma I_{2}}{1+I_{2}} \\
& =2+\frac{|\Gamma|}{1+I_{2}}:=L^{2} .
\end{aligned}
$$

Let $u_{\varepsilon}$ be the minimizer of $m_{\Gamma, k}(\varepsilon)$ for $\varepsilon \in\left(0, \min \left\{\varepsilon_{1}, \varepsilon_{2}\right\}\right)$. Now we can obtain that there exists a sequence of points $\left\{y_{n}\right\} \equiv:\left\{y_{\varepsilon_{n}}\right\}$ in $\mathbb{R}^{2}$ such that most of the "mass" of $u_{n}^{2}(x) \equiv: u_{\varepsilon_{n}}^{2}(x)$ is contained in a ball of fixed size centered at $\left\{y_{n}\right\}$. Here and below, we note that $\varepsilon_{n} \rightarrow 0$ if and only if $n \rightarrow+\infty$. At the same time, in the following we may assume that, up to a subsequence, $u_{n} \rightarrow v$ in $H_{k}^{1}\left(\mathbb{R}^{2}\right)$ as $n \rightarrow+\infty$.

Lemma 2.5. There exist positive constants $R$ and $\beta$ and a sequence $\left\{y_{n}\right\}$ such that

$$
\liminf _{n \rightarrow+\infty} \int_{B_{R}\left(y_{n}\right)} u_{n}^{2}(x) \geq \beta>0 .
$$


Proof. We will do it by a contradiction argument. If not, for any $R>0$, there exists a sequence $u_{n} \equiv: u_{\varepsilon_{n}}$ such that

$$
\lim _{n \rightarrow+\infty} \sup _{y \in \mathbb{R}^{2}} \int_{B_{R}(y)} u_{n}^{2}(x)=0 .
$$

Then by Lions's Lemma (e.g., [15]) one has $\left\|u_{n}\right\|_{p} \rightarrow 0$ for any $p>2$. By the fact that $t^{2}-\ln \left(1+t^{2}\right) \leq C t^{4}$ for some $C>0$, we have

$$
\begin{aligned}
H_{\varepsilon_{n}}\left(u_{n}\right) & =\int_{\mathbb{R}^{2}}\left|\nabla u_{n}\right|^{2}+\Gamma\left[u_{n}^{2}-\ln \left(1+\frac{u_{n}^{2}}{1+I\left(\varepsilon_{n} x\right)}\right)\right] \\
& =\int_{\mathbb{R}^{2}}\left|\nabla u_{n}\right|^{2}+\Gamma \int_{\mathbb{R}^{2}} \frac{I\left(\varepsilon_{n} x\right)}{1+I\left(\varepsilon_{n} x\right)} u_{n}^{2}+\Gamma \int_{\mathbb{R}^{2}}\left[\frac{u_{n}^{2}}{1+I\left(\varepsilon_{n} x\right)}-\ln \left(1+\frac{u_{n}^{2}}{1+I\left(\varepsilon_{n} x\right)}\right)\right] \\
& \geq \int_{\mathbb{R}^{2}}\left|\nabla u_{n}\right|^{2}+\frac{\Gamma I_{2}}{1+I_{2}}+o_{n}(1) .
\end{aligned}
$$

Therefore, by extracting a further subsequence if necessary, we can define $\Xi$ as

$$
\Xi=\lim _{n \rightarrow \infty} H_{\varepsilon_{n}}\left(u_{n}\right) .
$$

In view of Lemma 2.2, we have $k m\left(\frac{1}{k}, I_{2}\right) \geq \Xi \geq \frac{\Gamma I_{2}}{1+I_{2}}$, a contradiction to (2.1).

Lemma 2.6. If the sequence $\left\{y_{n}\right\} \subset \mathbb{R}^{2}$ obtained in Lemma 2.5 is bounded, and $u_{n} \rightarrow v$ in $H_{k}^{1}\left(\mathbb{R}^{2}\right)$ as $n \rightarrow+\infty$, then $\|v\|_{2}=1$.

Proof. Without loss of generality, we may assume that $y_{n} \equiv 0$. By Lemma 2.5, we know that there exist positive constants $R$ and $\beta$ such that

$$
\liminf _{n \rightarrow+\infty} \int_{B_{R}(0)} u_{n}^{2}(x) \geq \beta>0 .
$$

Since $u_{n} \rightarrow v$ in $H_{k}^{1}\left(\mathbb{R}^{2}\right)$ as $n \rightarrow+\infty$, by (2.4), we have

$$
\int_{B_{R}(0)} v^{2}(x) \geq \beta>0 .
$$

Thus, $v \neq 0$ and $u_{n} \rightarrow v$ in $L_{l o c}^{2}\left(\mathbb{R}^{2}\right)$ as $n \rightarrow+\infty$.

Therefore, $u_{n}(x) \rightarrow v(x)$ in $H^{1}\left(\mathbb{R}^{2}\right)$ and $u_{n} \rightarrow v$ in $L^{2}\left(B_{R}(0)\right)$. Hence, up to a subsequence, we may assume that $u_{n}(x) \rightarrow v(x)$ a.e. in $B_{R}(0)$. In view of (2.5), $v \neq 0$ in $B_{R}(0)$. By (2.5) there exists $\sigma>0$ such that

$$
u_{n}(x) \rightarrow v(x) \text { in } \Omega,
$$

where

$$
\Omega=\left\{x:|v(x)| \geq \sigma, x \in B_{R}(0)\right\} \subset B_{R}(0)
$$

and

$$
\mu(\Omega)>0 .
$$

Here $\mu(\Omega)$ is the Lebesgue measure of $\Omega$.

Now we assert that $\|v\|_{2}=1$. We argue it by contradiction, assuming that $A=\|v\|_{2}^{2} \in(0,1)$. We get a contradiction as follows. For the sake of convenience, we write $\tilde{v}(x)=\frac{v(x)}{\sqrt{1+I\left(\varepsilon_{n} x\right)}}$ and $\tilde{u}_{n}=\frac{u_{n}-v}{\sqrt{1+I\left(\varepsilon_{n} x\right)}}$. Then, by (2.6) and (2.7), we have

$$
\tilde{v}^{2}(x)=\frac{v^{2}(x)}{1+I\left(\varepsilon_{n} x\right)} \geq \frac{\sigma^{2}}{1+I_{2}}>0 \text { in } \Omega \text { for } \forall n
$$

and

$$
\tilde{u}_{n}^{2}(x)=\frac{\left(u_{n}(x)-v(x)\right)^{2}}{1+I\left(\varepsilon_{n} x\right)} \leq \frac{\left(u_{n}(x)-v(x)\right)^{2}}{1+I_{1}} \rightarrow 0 \text { in } \Omega \text { as } n \rightarrow+\infty .
$$


Let $f(s)=s-\ln (1+s), s \geq 0$. By (2.8) and (2.9), using Lemma 5.2 in [14], we can find $\alpha>0$ independent of $n$ such that

$$
\int_{\Omega} f\left(A\left(\frac{\tilde{v}^{2}}{A}\right)+(1-A) \frac{\tilde{u}_{n}^{2}}{\left\|u_{n}-v\right\|_{2}^{2}}\right) \leq-\alpha+\int_{\Omega} A f\left(\frac{\tilde{v}^{2}}{A}\right)+(1-A) f\left(\frac{\tilde{u}_{n}^{2}}{\left\|u_{n}-v\right\|_{2}^{2}}\right),
$$

as $n \rightarrow \infty$.

Then using the convexity of $f(s)=s-\ln (1+s), s \geq 0$ and (2.10), we have

$$
\begin{aligned}
m_{\Gamma, k}\left(\varepsilon_{n}\right)=H_{\varepsilon_{n}}\left(u_{n}\right) & \\
= & \int_{\mathbb{R}^{2}}\left|\nabla u_{n}\right|^{2}+\Gamma\left[u_{n}^{2}-\ln \left(1+\frac{u_{n}^{2}}{1+I\left(\varepsilon_{n} x\right)}\right)\right] \\
= & \int_{\mathbb{R}^{2}}\left|\nabla\left(v+\left(u_{n}-v\right)\right)^{2}\right|+\Gamma\left[\left(v+\left(u_{n}-v\right)\right)^{2}-\ln \left(1+\frac{\left(v+\left(u_{n}-v\right)\right)^{2}}{1+I\left(\varepsilon_{n} x\right)}\right)\right] \\
= & \int_{\mathbb{R}^{2}} A\left|\nabla\left(v /\|v\|_{2}\right)\right|^{2}+(1-A)\left|\nabla\left(\left(u_{n}-v\right) /\left\|u_{n}-v\right\|_{2}\right)\right|^{2} \\
& +\Gamma \int_{\mathbb{R}^{2}} I\left(\varepsilon_{n} x\right)\left(\tilde{v}^{2}+\tilde{u}_{n}^{2}\right)+\Gamma \int_{\mathbb{R}^{2}} f\left(A \frac{\tilde{v}^{2}}{A}+(1-A) \frac{\tilde{u}_{n}^{2}}{\left\|u_{n}-v\right\|_{2}^{2}}\right)+o_{\varepsilon_{n}}(1) \\
\geq & \int_{\mathbb{R}^{2}} A\left|\nabla\left(v /\|v\|_{2}\right)\right|^{2}+(1-A)\left|\nabla\left(\left(u_{n}-v\right) /\left\|u_{n}-v\right\|_{2}\right)\right|^{2} \\
& +\Gamma \int_{\mathbb{R}^{2}} I\left(\varepsilon_{n} x\right)\left(\tilde{v}^{2}+\tilde{u}_{n}^{2}\right)+\Gamma \int_{\mathbb{R}^{2}}\left(A f\left(\frac{\tilde{v}^{2}}{A}\right)+(1-A) f\left(\frac{\tilde{u}_{n}^{2}}{\left\|u_{n}-v\right\|_{2}^{2}}\right)\right) d x-\Gamma \alpha+o_{\varepsilon_{n}}(1) \\
= & A H_{\varepsilon_{n}}\left(\frac{v}{\|v\|_{2}}\right)+(1-A) H_{\varepsilon_{n}}\left(\frac{u_{n}-v}{\left\|u_{n}-v\right\|_{2}}\right)-\Gamma \alpha+o_{\varepsilon_{n}}(1) \\
\geq & A m_{\Gamma, k}\left(\varepsilon_{n}\right)+(1-A) m_{\Gamma, k}\left(\varepsilon_{n}\right)-\Gamma \alpha+o_{\varepsilon_{n}}(1) .
\end{aligned}
$$

In particular, by (2.11), for sufficiently small $\varepsilon_{n}$ satisfying $\left|o_{\varepsilon_{n}}(1)\right| \leq-\frac{\Gamma \alpha}{2}$, we have

$$
\begin{aligned}
m_{\Gamma, k}\left(\varepsilon_{n}\right) & \geq m_{\Gamma, k}\left(\varepsilon_{n}\right)-\Gamma \alpha+o_{\varepsilon_{n}}(1) \\
& \geq m_{\Gamma, k}\left(\varepsilon_{n}\right)-\frac{\Gamma \alpha}{2},
\end{aligned}
$$

a contradiction. Therefore, we have showed $\|v\|_{2}=1$ and Lemma 2.6 is proved.

Proposition 2.1. The sequence $\left\{y_{n}\right\}$ obtained in Lemma 2.5 satisfies $\left|y_{n}\right| \rightarrow+\infty$ as $n \rightarrow+\infty$.

Proof. Suppose for the contrary, there exist a constant $C>0$ and a subsequence of $\left\{y_{n}\right\}$, still denoted by $\left\{y_{n}\right\}$, such that

$$
\left|y_{n}\right| \leq C \text {. }
$$

We may assume without loss of generality that $y_{n} \equiv 0$.

According to Lemma 2.4, $\left\{u_{n}(x)\right\}$ is bounded in $H_{k}^{1}\left(\mathbb{R}^{2}\right)$. Then, passing to a subsequence if necessary, we have that $u_{n} \rightarrow v$ in $H_{k}^{1}\left(\mathbb{R}^{2}\right)$ as $n \rightarrow+\infty$.

By Lemma 2.6, we know that

$$
\|v\|_{2}=1
$$

Since $u_{n}$ satisfies

$$
-\Delta u_{n}+\Gamma \frac{I\left(\varepsilon_{n} x\right)+u_{n}^{2}}{1+I\left(\varepsilon_{n} x\right)+u_{n}^{2}} u_{n}=\lambda_{\varepsilon_{n}} u_{n}, x \in \mathbb{R}^{2},
$$

by the elliptic estimates to (2.13), we have

$$
-\Delta v+\Gamma \frac{I(0)+v^{2}}{1+I(0)+v^{2}} v=\lambda_{\Gamma} v, x \in \mathbb{R}^{2} .
$$


Here we have used Lemma 2.3 which implies $\lambda_{\varepsilon_{n}} \rightarrow \lambda_{\Gamma}<0$ as $\varepsilon_{n} \rightarrow 0$ (up to a subsequence). Since $v \geq 0$ satisfying (2.14) depends on $\Gamma$, we denote $v$ as $v_{\Gamma}$. Rewriting (2.14) as

$$
-\Delta v_{\Gamma}+d(x) v_{\Gamma}=0, x \in \mathbb{R}^{2},
$$

with $d(x)=\Gamma \frac{I(0)+v_{\Gamma}^{2}}{1+I(0)+v_{\Gamma}^{2}}-\lambda_{\Gamma}$. Moreover,

$$
d(x)^{-}=\lambda_{\Gamma}-\Gamma\left(\frac{I(0)+v_{\Gamma}^{2}}{1+I(0)+v_{\Gamma}^{2}}\right)^{+} \leq|\Gamma|, x \in \mathbb{R}^{2},
$$

where $u^{+}=\max \{u, 0\}, u^{-}=\max \{-u, 0\}$.

Now we will prove that there exists $M=M(\Gamma)>0$ depending only on $\Gamma$, such that

$$
\left\|v_{\Gamma}\right\|_{\infty}=\left\|v_{\Gamma}\right\|_{L^{\infty}\left(\mathbb{R}^{2}\right)} \leq M
$$

In particular, $\left\|v_{\Gamma}\right\|_{\infty}$ is independent of $I(0)$.

To prove (2.17), we need the following lemma.

Lemma 2.7. (Subsolution estimate, Theorem C.1.2 of [16])

Suppose $u \in H^{1}\left(B_{2}\left(x_{0}\right)\right)$ solves

$$
-\Delta u+V(x) u=0 \quad \text { in } B_{2}\left(x_{0}\right) .
$$

Then

$$
\left|u\left(x_{0}\right)\right| \leq C \int_{B_{1}\left(x_{0}\right)}|u|,
$$

where $C>0$ is a constant depending only on the following quantities:

$$
\begin{aligned}
& \sup _{x \in B_{1}\left(x_{0}\right)} \int_{|y-x| \leq 1} V(y)^{-} d y \quad \text { if } N=1 ; \\
& \sup _{x \in B_{1}\left(x_{0}\right)} \int_{|y-x| \leq \frac{1}{2}} \ln \left(|x-y|^{-1}\right) V(y)^{-} d y \quad \text { if } N=2 ; \\
& \sup _{x \in B_{1}\left(x_{0}\right)} \int_{|y-x| \leq 1}|x-y|^{2-N} V(y)^{-} d y \quad \text { if } N \geq 3 .
\end{aligned}
$$

By (2.16), for $N=2$, we have

$$
\begin{aligned}
\int_{|y-x| \leq \frac{1}{2}} \ln \left(|x-y|^{-1}\right) d(y)^{-} d y & \leq|\Gamma| \int_{|y-x| \leq \frac{1}{2}} \ln \left(|x-y|^{-1}\right) d y \\
& \leq|\Gamma| \int_{|z| \leq \frac{1}{2}} \ln \left(|z|^{-1}\right) d z \\
& \leq C_{1}|\Gamma| .
\end{aligned}
$$

Here $C_{1}$ does not depend on $\Gamma$.

Therefore, by Lemma 2.7 and (2.18), we obtain

$$
\left|v_{\Gamma}\left(x_{0}\right)\right| \leq C \int_{B_{1}\left(x_{0}\right)}\left|v_{\Gamma}(y)\right| d y \text { for } x_{0} \in \mathbb{R}^{2},
$$

where $C=C(\Gamma)>0$ is a constant depending only on $\Gamma$. In view of $\int_{\mathbb{R}^{2}}\left|v_{\Gamma}(y)\right|^{2} d y=1$, by (2.19), we know that there exists $M=M(\Gamma)>0$ depending only on $\Gamma$, such that

$$
\left|v_{\Gamma}\left(x_{0}\right)\right| \leq M \text { for } x_{0} \in \mathbb{R}^{2} .
$$


Thus, the proof of (2.17) is complete.

Meanwhile, we have

$$
\begin{aligned}
\lim _{\varepsilon_{n} \rightarrow 0} H_{\varepsilon_{n}}\left(u_{n}\right) & =\lim _{\varepsilon_{n} \rightarrow 0} \int_{\mathbb{R}^{2}}\left|\nabla u_{n}\right|^{2}+\Gamma\left[u_{n}^{2}-\ln \left(1+\frac{u_{n}^{2}}{1+I\left(\varepsilon_{n} x\right)}\right)\right] \\
& \geq \inf _{v \in H_{k}^{1}\left(\mathbb{R}^{2}\right), \int_{\mathbb{R}^{2}} v^{2}=1} \int_{\mathbb{R}^{2}}|\nabla v|^{2}+\Gamma\left[v^{2}-\ln \left(1+\frac{v^{2}}{1+I(0)}\right)\right] .
\end{aligned}
$$

Now we will prove

Assertion 2.1.

$$
\inf _{v \in H_{k}^{1}\left(\mathbb{R}^{2}\right), \int_{\mathbb{R}^{2}} v^{2}=1} \int_{\mathbb{R}^{2}}|\nabla v|^{2}+\Gamma\left[v^{2}-\ln \left(1+\frac{v^{2}}{1+I(0)}\right)\right]>k m\left(\frac{1}{k}, I_{2}\right) .
$$

Postponing the proof of Assertion 2.1, we finish the proof of Proposition 2.1. If Assertion 2.1 holds, by (2.21), we have

$$
\lim _{\varepsilon_{n} \rightarrow 0} H_{\varepsilon_{n}}\left(u_{n}\right)>k m\left(\frac{1}{k}, I_{2}\right),
$$

which is a contradiction to Lemma 2.2. Therefore, the previous assumption (2.12) is false, and we obtain the conclusions of Proposition 2.1.

We now return to the proof for Assertion 2.1.

Firstly, we note that

Lemma 2.8. For given $T>0$, there exists $\alpha_{0}>0$, such that

$$
1+\frac{y}{1+I(0)}>e^{\frac{y}{1+I_{2}}}, \text { for } 0<y \leq T
$$

as long as $0<1+I(0)<\alpha_{0}$.

The proof of Lemma 2.8 is elementary, we omit it here.

For given $k \in \mathbb{N}^{+}$, it is easy to see

$$
e^{\frac{y}{1+I_{2}}} \geq\left(1+\frac{y}{k\left(1+I_{2}\right)}\right)^{k} \text { for } 0<y \leq M^{2} .
$$

Then, by Lemma 2.8, for $T=M^{2}$, there exists $\alpha_{0}=\alpha_{0}(\Gamma)>0$, such that

$$
1+\frac{y}{1+I(0)}>\left(1+\frac{y}{k\left(1+I_{2}\right)}\right)^{k} \text { for } 0<y \leq M^{2}
$$

if $0<1+I(0)<\alpha_{0}$.

Since $0<v_{\Gamma}(x) \leq M$ for $x \in \mathbb{R}^{2}$, by (2.23), we have

$$
\int_{\mathbb{R}^{2}} \ln \left(1+\frac{v_{\Gamma}^{2}}{1+I(0)}\right)>\int_{\mathbb{R}^{2}} \ln \left(1+\frac{v_{\Gamma}^{2}}{k\left(1+I_{2}\right)}\right)^{k} .
$$

On the other hand, since $v_{\Gamma}$ satisfies (2.14) and $\int_{\mathbb{R}^{2}} v_{\Gamma}^{2}=1$, denoting $\widetilde{v_{\Gamma}}=\frac{v_{\Gamma}}{\sqrt{k}}$, we have $\int_{\mathbb{R}^{2}}{\widetilde{v_{\Gamma}}}^{2}=\frac{1}{k}$ and

$$
\begin{aligned}
k \int_{\mathbb{R}^{2}} \frac{\left|\nabla v_{\Gamma}\right|^{2}}{k}+k \Gamma \int_{\mathbb{R}^{2}}\left[\frac{v_{\Gamma}^{2}}{k}-\ln \left(1+\frac{v_{\Gamma}^{2}}{k\left(1+I_{2}\right)}\right)\right] & =k \int_{\mathbb{R}^{2}}\left|\nabla \widetilde{v_{\Gamma}}\right|^{2}+\Gamma\left[{\widetilde{v_{\Gamma}}}^{2}-\ln \left(1+\frac{{\widetilde{v_{\Gamma}}}^{2}}{\left(1+I_{2}\right)}\right)\right] \\
& \geq k m\left(\frac{1}{k}, I_{2}\right) .
\end{aligned}
$$


Consequently, by (2.25) and (2.24), we obtain

$$
\begin{aligned}
& \inf _{v \in H_{k}^{1}\left(\mathbb{R}^{2}\right), \int_{\mathbb{R}^{2}} v^{2}=1} \int_{\mathbb{R}^{2}}|\nabla v|^{2}+\Gamma\left[v^{2}-\ln \left(1+\frac{v^{2}}{1+I(0)}\right)\right] \\
& =\int_{\mathbb{R}^{2}}\left|\nabla v_{\Gamma}\right|^{2}+\Gamma\left[v_{\Gamma}^{2}-\ln \left(1+\frac{v_{\Gamma}^{2}}{1+I(0)}\right)\right] \\
& >\int_{\mathbb{R}^{2}}\left|\nabla v_{\Gamma}\right|^{2}+\Gamma\left[v_{\Gamma}^{2}-\ln \left(1+\frac{v_{\Gamma}^{2}}{k\left(1+I_{2}\right)}\right)^{k}\right] \\
& =k \int_{\mathbb{R}^{2}} \frac{\left|\nabla v_{\Gamma}\right|^{2}}{k}+k \Gamma \int_{\mathbb{R}^{2}}\left[\frac{v_{\Gamma}^{2}}{k}-\ln \left(1+\frac{v_{\Gamma}^{2}}{k\left(1+I_{2}\right)}\right)\right] \\
& \geq k m\left(\frac{1}{k}, I_{2}\right) .
\end{aligned}
$$

Here we have used $\int_{\mathbb{R}^{2}} v_{\Gamma}^{2}=1$.

Hence, by (2.26), the proof of Assertion 2.1 is complete.

Lemma 2.9. Let $\varepsilon_{n} \rightarrow 0$ and $u_{n}(x) \in H_{k}^{1}\left(\mathbb{R}^{2}\right)$ satisfy $\int_{\mathbb{R}^{2}} u_{n}^{2}(x)=1$ and

$$
\varlimsup_{n \rightarrow+\infty} H_{\varepsilon_{n}}\left(u_{n}\right) \leq k m\left(\frac{1}{k}, I_{2}\right),
$$

where $u_{n}(x)$ is the minimizer of $m_{\Gamma, k}\left(\varepsilon_{n}\right)$. Then there exist a subsequence of $\left\{u_{n}(x)\right\}$ (still denoted by $\left.\left\{u_{n}(x)\right\}\right)$ and $\left\{y_{n}\right\}$ satisfying $\# G_{k}\left(y_{n}\right)=k$, such that for each $\gamma>0$ there exists $R=R(\gamma)>0$,

$$
\int_{B_{R}\left(y_{n}^{i}\right)} u_{n}^{2}(x) \geq \frac{1}{k}-\gamma,
$$

where $y_{n}^{i}=g_{i} y_{n}, g_{i} \in G_{k}, i=1,2, \ldots, k$ and $g_{k} y_{n}=y_{n}$.

Remark 2.1. The \# $G_{k}(x)$ in Lemma 2.9 stands for the cardinal number of $G_{k}(x)$, the orbit of $x$ under the action $G_{k}$.

Proof. We only consider $k \geq 2$ (for $k=1$, this is the case of Lemma 3.1 in [9]). Applying the concentration compactness principle $[17,18]$, we get three possibilities: vanishing, dichotomy and compactness. Vanishing can be ruled out by using Lemma 2.5. If compactness happens, there exists a subsequence of $\left\{u_{n}(x)\right\}$ (still denoted by $\left.\left\{u_{n}(x)\right\}\right)$, and $\left\{y_{n}\right\}$ such that for any $\gamma>0$ there exists $R=R(\gamma)>0$ with the property that

$$
\int_{B_{R}\left(y_{n}\right)} u_{n}^{2}(x) \geq 1-\gamma .
$$

Now we can also get a contradiction as follows. Firstly, we claim that there exists $R_{0}>0$ such that $\left\{y_{n}\right\}$ in (2.27) satisfying

$$
\left|y_{n}\right| \leq R_{0}
$$

If (2.28) is not true, then for a subsequence $\left|y_{n}\right| \rightarrow+\infty$ as $n \rightarrow+\infty$. By the symmetry of $\mathbb{R}^{2}$ and $u_{n}\left(g^{-1} x\right)=$ $u_{n}(x)$ with $g \in G_{k}$, we have

$$
\int_{\mathbb{R}^{2}} u_{n}^{2}(x) \geq \int_{\cup_{i=1}^{k} B_{R}\left(y_{n}^{i}\right)} u_{n}^{2}(x) \geq k-k \gamma .
$$

This is a contradiction with $\int_{\mathbb{R}^{2}} u_{n}^{2}(x)=1$.

On the other hand, by Proposition 2.1, we know that $\left\{y_{n}\right\}$ in (2.27) satisfying $\left|y_{n}\right| \rightarrow+\infty$ as $n \rightarrow+\infty$, which produces a contradiction with (2.28). Therefore, compactness does not happen. 
With vanishing and compactness both being ruled out, we obtain dichotomy of the sequence. Now by Proposition 2.1, we note that the orbit of $\left\{y_{n}\right\}$ under the action of $G_{k}$ contains exactly $k$ points:

$$
y_{n}^{1}, y_{n}^{2}, \ldots, y_{n}^{k-1}, y_{n}^{k}=y_{n}
$$

and the distance between any two of these $k$ points tends to infinity as $n \rightarrow \infty$. By the symmetry of the domain $\mathbb{R}^{2}$ and the fact that $u_{n}(x)$ are $G$-invariant, one obtains for any fixed $n$ sufficiently large,

$$
0<\int_{B_{R}\left(y_{n}^{i}\right)} u_{n}^{2}(x) \leq \frac{1}{k}, i=1,2, \ldots, k .
$$

Since $u_{n}\left(g_{i} x\right)=u_{n}(x), i=1,2, \ldots, k$, we have

$$
\int_{B_{R}\left(y_{n}^{i}\right)} u_{n}^{2}(x)=\int_{B_{R}\left(y_{n}^{j}\right)} u_{n}^{2}(x), i \neq j .
$$

Now we claim that for all $\gamma>0$, there exists $R=R(\gamma)>0$, such that

$$
\int_{B_{R}\left(y_{n}^{i}\right)} u_{n}^{2}(x) \geq \frac{1}{k}-\gamma, i=1,2, \ldots, k,
$$

as $n \rightarrow+\infty$. If not, we assume that there exists $\alpha$ satisfying $0<\alpha<\frac{1}{k}$, such that for all $\gamma>0$, there exists $R=R(\gamma)>0$,

$$
\left|\int_{B_{R}\left(y_{n}^{i}\right)} u_{n}^{2}(x)-\alpha\right| \leq \gamma, i=1,2, \ldots, k,
$$

as $n \rightarrow+\infty$. Then $A:=k \alpha<1$. We will derive a contradiction as follows.

Let

$$
w_{n, i}(x)=u_{n}\left(x+y_{n}^{i}\right), i=1,2, \ldots, k
$$

Then

$$
-\Delta w_{n, i}+\Gamma \frac{I\left(\varepsilon_{n} x+\varepsilon_{n} y_{n}^{i}\right)+w_{n, i}^{2}}{1+I\left(\varepsilon_{n} x+\varepsilon_{n} y_{n}^{i}\right)+w_{n, i}^{2}} w_{n, i}=\lambda_{\varepsilon_{n}} w_{n, i}, \quad x \in \mathbb{R}^{2} .
$$

By Lemma 2.5, for $0<\gamma<\frac{\alpha}{2}$, there exists $R=R(\gamma)>0$, such that

$$
\liminf _{n \rightarrow+\infty} \int_{B_{R}(0)} w_{n, i}^{2}(x) \geq \frac{\alpha}{2} \geq \beta>0, i=1,2, \ldots, k .
$$

From Lemma 2.4,

$$
w_{n, i} \text { is bounded in } H^{1}\left(\mathbb{R}^{2}\right), i=1,2, \ldots, k .
$$

Hence, $w_{n, i} \rightarrow w_{i}$ in $H^{1}\left(\mathbb{R}^{2}\right)$ and $w_{n, i} \rightarrow w_{i} \neq 0$ in $L^{2}\left(B_{R}(0)\right), i=1,2, \ldots, k$.

By (2.32), $\int_{B_{R}(0)} w_{i}^{2} \geq \beta>0, i=1,2, \ldots, k$. Therefore, there exists $\tau>0$ such that

$$
D_{i}=\left\{x: w_{i}(x) \geq \tau, x \in B_{R}(0)\right\} \subset B_{R}(0)
$$

and

$$
\mu\left(D_{i}\right)>0,
$$

where $\mu\left(D_{i}\right)$ denotes Lebesgue measure of $D_{i}, i=1,2, \ldots, k$.

Denoting

$$
D_{n}^{i}:=D_{i}+y_{n}^{i}=\left\{x+y_{n}^{i}: x \in D_{i}\right\}, i=1,2, \ldots, k,
$$

we have

$$
w_{i}\left(x+y_{n}^{i}\right) \geq \tau, x \in D_{i}, i=1,2, \ldots, k .
$$


Moreover, from the fact $w_{n, i} \rightarrow w_{i}$ in $L^{2}\left(B_{R}(0)\right)$, we have

$$
\lim _{n \rightarrow \infty} \int_{D_{i}}\left|w_{n, i}(x)-w_{i}(x)\right|^{2}=0 \text { and } w_{n, i}(x) \rightarrow w_{i}(x) \neq 0 \text { a.e. in } D_{i} .
$$

Then, by elliptic estimates we have $\max _{B_{R}(0)}\left|w_{n, i}(x)-w_{i}(x)\right| \rightarrow 0$ for $i=1,2, \ldots, k$ as $n \rightarrow \infty$. Since $\left|y_{n}^{i}\right| \rightarrow+\infty, i=1,2, \ldots, k$, as $n \rightarrow+\infty$ and $\left|y_{n}^{i}-y_{n}^{j}\right| \rightarrow+\infty, i \neq j$ as $n \rightarrow+\infty$, we have

$$
D_{n}^{i} \cap D_{n}^{j}=\emptyset, i \neq j \text {, }
$$

as $n \rightarrow+\infty$. Then, we get

$$
\lim _{n \rightarrow+\infty}\left\|\sum_{i=1}^{k} w_{i}\left(x-y_{n}^{i}\right)\right\|_{2}^{2}=\lim _{n \rightarrow+\infty} \sum_{i=1}^{k} \int_{\mathbb{R}^{2}} w_{i}^{2}\left(x-y_{n}^{i}\right)=k \alpha=A .
$$

Therefore, by (2.38) and Brezis-Lieb Lemma [19], we have

$$
1-A=\left\|u_{n}\right\|_{2}^{2}-\left\|\sum_{i=1}^{k} w_{i}\left(x-y_{n}^{i}\right)\right\|_{2}^{2}+o_{n}(1)=\left\|u_{n}-\sum_{i=1}^{k} \int_{\mathbb{R}^{2}} w_{i}\left(x-y_{n}^{i}\right)\right\|_{2}^{2}+o_{n}(1) .
$$

Let $f(s)=s-\ln (1+s)$. Based on (2.36) and the fact

$$
\max _{\cup_{i=1}^{k} D_{n}^{i}}\left|u_{n}-\sum_{i=1}^{k} w_{i}\left(x-y_{n}^{i}\right)\right| \leq \sum_{i=1}^{k} \max _{B_{R}(0)}\left|w_{n, i}(x)-w_{i}(x)\right| \rightarrow 0
$$

as $n \rightarrow \infty$, using Lemma 5.2 in [14] we can find $\delta>0$ such that

$$
\begin{aligned}
& \int_{\cup_{i=1}^{k} D_{n}^{i}} f\left(A \frac{\left(\sum_{i=1}^{k} w_{i}\left(x-y_{n}^{i}\right)\right)^{2}}{\left\|\sum_{i=1}^{k} w_{i}\left(x-y_{n}^{i}\right)\right\|_{2}^{2}\left(1+I\left(\varepsilon_{n} x\right)\right)}+(1-A) \frac{\left(u_{n}-\sum_{i=1}^{k} w_{i}\left(x-y_{n}^{i}\right)\right)^{2}}{\left\|u_{n}-\sum_{i=1}^{k} w_{i}\left(x-y_{n}^{i}\right)\right\|_{2}^{2}\left(1+I\left(\varepsilon_{n} x\right)\right)}\right) \\
\leq-\delta+ & \int_{\cup_{i=1}^{k} D_{n}^{i}} A f\left(\frac{\left(\sum_{i=1}^{k} w_{i}\left(x-y_{n}^{i}\right)\right)^{2}}{\left\|\sum_{i=1}^{k} w_{i}\left(x-y_{n}^{i}\right)\right\|_{2}^{2}\left(1+I\left(\varepsilon_{n} x\right)\right)}\right)+\int_{\cup_{i=1}^{k} D_{n}^{i}}(1-A) f\left(\frac{\left(u_{n}-\sum_{i=1}^{k} w_{i}\left(x-y_{n}^{i}\right)\right)^{2}}{\left\|u_{n}-\sum_{i=1}^{k} w_{i}\left(x-y_{n}^{i}\right)\right\|_{2}^{2}\left(1+I\left(\varepsilon_{n} x\right)\right)}\right)
\end{aligned}
$$

as $n \rightarrow \infty$.

Thus, using the above facts we have

$$
\begin{aligned}
& m_{\Gamma, k}\left(\varepsilon_{n}\right)=H_{\varepsilon_{n}}\left(u_{n}\right)=\int_{\mathbb{R}^{2}}\left|\nabla u_{n}\right|^{2}+\Gamma\left[u_{n}^{2}-\ln \left(1+\frac{u_{n}^{2}}{1+I\left(\varepsilon_{n} x\right)}\right)\right] \\
& =\int_{\cup_{i=1}^{k} B_{R}\left(y_{n}^{i}\right)}\left|\nabla u_{n}\right|^{2}+\int_{\mathbb{R}^{2} \mid \cup_{i=1}^{k} B_{R}\left(y_{n}^{i}\right)}\left|\nabla u_{n}\right|^{2}+\Gamma \int_{\cup_{i=1}^{k} B_{R}\left(y_{n}^{i}\right)} \frac{I\left(\varepsilon_{n} x\right) u_{n}^{2}}{1+I\left(\varepsilon_{n} x\right)} \\
& \quad+\Gamma \int_{\mathbb{R}^{2} \mid \cup_{i=1}^{k} B_{R}\left(y_{n}^{i}\right)} \frac{I\left(\varepsilon_{n} x\right) u_{n}^{2}}{1+I\left(\varepsilon_{n} x\right)}+\Gamma \int_{\mathbb{R}^{2}}\left[\frac{u_{n}^{2}}{1+I\left(\varepsilon_{n} x\right)}-\ln \left(1+\frac{u_{n}^{2}}{1+I\left(\varepsilon_{n} x\right)}\right)\right] \\
& =\int_{\mathbb{R}^{2}} A\left|\frac{\nabla\left(\sum_{i=1}^{k} w_{i}\left(x-y_{n}^{i}\right)\right)}{\left\|\sum_{i=1}^{k} w_{i}\left(x-y_{n}^{i}\right)\right\|_{2}}\right|^{2}+(1-A)\left|\frac{\nabla\left(u_{n}-\sum_{i=1}^{k} w_{i}\left(x-y_{n}^{i}\right)\right)}{\left\|u_{n}-\sum_{i=1}^{k} w_{i}\left(x-y_{n}^{i}\right)\right\|_{2}}\right|^{2} \\
& +\Gamma \int_{\mathbb{R}^{2}} A \frac{I\left(\varepsilon_{n} x\right)\left(\sum_{i=1}^{k} w_{i}\left(x-y_{n}^{i}\right)\right)^{2}}{\left\|\sum_{i=1}^{k} w_{i}\left(x-y_{n}^{i}\right)\right\|_{2}^{2}\left(1+I\left(\varepsilon_{n} x\right)\right)}+(1-A) \frac{I\left(\varepsilon_{n} x\right)\left(u_{n}-\sum_{i=1}^{k} w_{i}\left(x-y_{n}^{i}\right)\right)^{2}}{\left\|u_{n}-\sum_{i=1}^{k} w_{i}\left(x-y_{n}^{i}\right)\right\|_{2}^{2}\left(1+I\left(\varepsilon_{n} x\right)\right)} \\
& +\Gamma \int_{\mathbb{R}^{2}} f\left(A \frac{\left(\sum_{i=1}^{k} w_{i}\left(x-y_{n}^{i}\right)\right)^{2}}{\left\|\sum_{i=1}^{k} w_{i}\left(x-y_{n}^{i}\right)\right\|_{2}^{2}\left(1+I\left(\varepsilon_{n} x\right)\right)}+(1-A) \frac{\left(u_{n}-\sum_{i=1}^{k} w_{i}\left(x-y_{n}^{i}\right)\right)^{2}}{\left\|u_{n}-\sum_{i=1}^{k} w_{i}\left(x-y_{n}^{i}\right)\right\|_{2}^{2}\left(1+I\left(\varepsilon_{n} x\right)\right)}\right)+o_{n}(1) \\
& \geq A H_{\varepsilon_{n}}\left(\frac{\sum_{i=1}^{k} w_{i}\left(x-y_{n}^{i}\right)}{\left\|\sum_{i=1}^{k} w_{i}\left(x-y_{n}^{i}\right)\right\|_{2}}\right)+(1-A) H_{\varepsilon_{n}}\left(\frac{u_{n}-\sum_{i=1}^{k} w_{i}\left(x-y_{n}^{i}\right)}{\left\|u_{n}-\sum_{i=1}^{k} w_{i}\left(x-y_{n}^{i}\right)\right\|_{2}}\right)-\Gamma \delta+o_{n}(1) \\
& \geq A m_{\Gamma, k}\left(\varepsilon_{n}\right)+(1-A) m_{\Gamma, k}\left(\varepsilon_{n}\right)-\Gamma \delta+o_{n}(1) .
\end{aligned}
$$


Sending $n \rightarrow \infty$ we obtain a contradiction.

Hence, we conclude that $\alpha=\frac{1}{k}$.

As a result, there exist a subsequence $\left\{u_{n}(x)\right\}$ and $\left\{y_{n}\right\}$, such that for each $\gamma>0$ there exists $R=R(\gamma)>0$,

$$
\int_{B_{R}\left(y_{n}^{i}\right)} u_{n}^{2}(x) \geq \frac{1}{k}-\gamma,
$$

where $y_{n}^{i}=g_{i} y_{n}, g_{i} \in G_{k}, i=1,2, \ldots, k$ and $g_{k} y_{n}=y_{n}$.

\section{Proof of Theorem 1.1}

In this section, we will give the proof for the conclusions (i) and (ii) of Theorem 1.1 as $\varepsilon \rightarrow 0^{+}$. To be more specific, the conclusions of (i) in Theorem 1.1 will be proved by Lemma 3.1, and the conclusions of (ii) in Theorem 1.1 will be proved by Lemma 3.2 and Lemma 3.3.

\section{Lemma 3.1.}

$$
\lim _{\varepsilon \rightarrow 0^{+}} m_{\Gamma, k}(\varepsilon)=k m\left(\frac{1}{k}, I_{2}\right) .
$$

Proof. By Lemma 3.1 in [9], we have $\lim _{\varepsilon \rightarrow 0} m_{\Gamma, 1}(\varepsilon)=m\left(1, I_{2}\right)$. Now in the following, we always assume $k \geq 2$. Suppose the conclusion is not true, by Lemma 2.2, we know that there exist $\varepsilon_{n} \rightarrow 0$ and $u_{n}(x) \in H_{k}^{1}\left(\mathbb{R}^{2}\right)$ a solution of (1.2) with $\int_{\mathbb{R}^{2}} u_{n}^{2}(x)=1$, such that

$$
\lim _{\varepsilon_{n} \rightarrow 0} m_{\Gamma, k}\left(\varepsilon_{n}\right)=\lim _{\varepsilon_{n} \rightarrow 0} H_{\varepsilon_{n}}\left(u_{n}\right)=B<k m\left(\frac{1}{k}, I_{2}\right) .
$$

On the other hand, by Lemma 2.9, for each $\gamma>0$, there exists $R=R(\gamma)>0$, such that

$$
\int_{B_{R}\left(y_{n}^{i}\right)} u_{n}^{2}(x) \geq \frac{1}{k}-\gamma, i=1,2, \ldots, k .
$$

Let $\eta=\eta(t)$ be a smooth nonincreasing function on $[0,+\infty)$ such that $\eta(t)=1$, for $t \in[0,1], \eta(t)=0$, for $t \geq 2$, and $\left|\eta^{\prime}(t)\right| \leq 2$. Setting

$$
\overline{w_{n, i}}(x)=\eta\left(\frac{x-y_{n}^{i}}{R}\right) u_{n}(x), x \in \mathbb{R}^{2},
$$

then $\overline{w_{n, i}}(x) \in H^{1}\left(\mathbb{R}^{2}\right)$. By choosing $R$ large enough (for fixed $\gamma>0$ ), we may assume

$$
\frac{1}{k} \geq \int_{\mathbb{R}^{2}}{\overline{w_{n, i}}}^{2}(x) \geq \int_{B_{R}\left(y_{n}^{i}\right)}{\overline{w_{n, i}}}^{2}(x) \geq \frac{1}{k}-2 \gamma, i=1,2, \ldots, k .
$$


Therefore, we have

$$
\begin{aligned}
E_{\varepsilon_{n}}\left(u_{n}\right) & =\int_{\mathbb{R}^{2}}\left|\nabla u_{n}\right|^{2}+\Gamma\left[u_{n}^{2}-\ln \left(1+\frac{u_{n}^{2}}{1+I\left(\varepsilon_{n} x\right)}\right)\right] \\
& =\int_{\cup_{i=1}^{k} B_{R}\left(y_{n}^{i}\right)}\left|\nabla u_{n}\right|^{2}+\Gamma\left[u_{n}^{2}-\ln \left(1+\frac{u_{n}^{2}}{1+I\left(\varepsilon_{n} x\right)}\right)\right]+o_{\gamma}(1) \\
& \geq \int_{\cup_{i=1}^{k} B_{R}\left(y_{n}^{i}\right)}\left|\nabla u_{n}\right|^{2}+\Gamma\left[u_{n}^{2}-\ln \left(1+\frac{u_{n}^{2}}{1+I_{2}}\right)\right]+o_{\gamma}(1) \\
& =\sum_{i=1}^{k} \int_{\mathbb{R}^{2}}\left|\nabla \bar{w}_{n, i}(x)\right|^{2}+\Gamma\left[\bar{w}_{n, i}{ }^{2}(x)-\ln \left(1+\frac{w_{n, i}}{1+I_{2}}\right)\right]+o_{\gamma}(1) \\
& \geq k \inf _{w \in H^{1}\left(\mathbb{R}^{2}\right), \int_{\mathbb{R}^{2}} w^{2}=\frac{1}{k}} \int_{\mathbb{R}^{2}}|\nabla w|^{2}+\Gamma\left[w^{2}-\ln \left(1+\frac{w^{2}}{1+I_{2}}\right)\right]+o_{\gamma}(1) \\
& =k m\left(\frac{1}{k}, I_{2}\right)+o_{\gamma}(1) .
\end{aligned}
$$

Letting $\varepsilon_{n} \rightarrow 0$ and $\gamma \rightarrow 0$, we have

$$
B \geq k m\left(\frac{1}{k}, I_{2}\right),
$$

a contradiction to (3.1).

Lemma 3.2. The sequence $\left\{y_{n}\right\}$ obtained in Lemma 2.5 satisfies

$$
\varepsilon_{n}\left|y_{n}\right| \rightarrow\left|e_{1}\right|=1 \text { as } n \rightarrow+\infty \text {, where } e_{1} \in S^{1} .
$$

Proof. Suppose on the contrary that there exists a sequence $\varepsilon_{n}$ such that $\left|\varepsilon_{n} y_{\varepsilon_{n}}\right| \rightarrow+\infty$ as $n \rightarrow+\infty$. By (B1), we have $I_{2}=\max _{x \in \mathbb{R}^{2}} I(x)>\limsup _{|x| \rightarrow+\infty} I(x)=I_{\infty} \geq I_{1}>-1$, then there exists $\delta>0$, such that

$$
I\left(\varepsilon_{n} x+\varepsilon_{n} y_{n}\right)<I_{2}-\delta \text { and } 1+I_{2}-\delta>0
$$

for $n$ large enough and $\left|\varepsilon_{n} x\right| \leq 1$.

Accordingly, under the action of $G_{k}$, we have

$$
I\left(\varepsilon_{n} X+\varepsilon_{n} y_{n}^{i}\right)<I_{2}-\delta, i=1,2, \ldots, k \text { and } 1+I_{2}-\delta>0
$$

for $n$ large enough and $\left|\varepsilon_{n} x\right| \leq 1$. 
Let $\eta=\eta(t)$ be the cut-off function used in Lemma 3.1. Also, we define $\widetilde{w_{n, i}}(x)=\eta\left(\frac{x}{R}\right) w_{n, i}(x)=\eta\left(\frac{x}{R}\right) u_{n}(x+$ $\left.y_{n}^{i}\right), x \in \mathbb{R}^{2}$. Then we have

$$
\begin{aligned}
E_{\varepsilon_{n}}\left(u_{n}\right) & =\int_{\mathbb{R}^{2}}\left|\nabla u_{n}\right|^{2}+\Gamma\left[u_{n}^{2}-\ln \left(1+\frac{u_{n}^{2}}{1+I\left(\varepsilon_{n} x\right)}\right)\right] \\
& =\int_{\cup_{i=1}^{k} B_{R}\left(y_{n}^{i}\right)}\left|\nabla u_{n}\right|^{2}+\Gamma\left[u_{n}^{2}-\ln \left(1+\frac{u_{n}^{2}}{1+I\left(\varepsilon_{n} x\right)}\right)\right]+o_{\gamma}(1) \\
& =\sum_{i=1}^{k} \int_{B_{R}(0)}\left|\nabla u_{n}\left(x+y_{n}^{i}\right)\right|^{2}+\Gamma\left[u_{n}^{2}\left(x+y_{n}^{i}\right)-\ln \left(1+\frac{u_{n}^{2}\left(x+y_{n}^{i}\right)}{1+I\left(\varepsilon_{n} x+\varepsilon_{n} y_{n}^{i}\right)}\right)\right]+o_{\gamma}(1) \\
& =\sum_{i=1}^{k} \int_{\mathbb{R}^{2}}\left|\nabla \widetilde{w_{n, i}}(x)\right|^{2}+\Gamma\left[{\widetilde{w_{n}, i}}^{2}(x)-\ln \left(1+\frac{{\widetilde{w_{n, i}}}^{2}(x)}{1+I\left(\varepsilon_{n} x+\varepsilon_{n} y_{n}^{i}\right)}\right)\right]+o_{\gamma}(1) \\
& =\sum_{i=1}^{k} \int_{\mathbb{R}^{2} \cap\left\{|x| \leq \frac{1}{\varepsilon_{n}}\right\}}\left|\nabla \widetilde{w_{n, i}}(x)\right|^{2}+\Gamma\left[{\widetilde{w_{n, i}}}^{2}(x)-\ln \left(1+\frac{{\widetilde{w_{n, i}}}^{2}(x)}{1+I\left(\varepsilon_{n} x+\varepsilon_{n} y_{n}^{i}\right)}\right)\right] \\
& +\sum_{i=1}^{k} \int_{\mathbb{R}^{2} \cap\left\{|x| \geq \frac{1}{\varepsilon_{n}}\right\}}\left|\nabla \widetilde{w_{n, i}}(x)\right|^{2}+\Gamma\left[{\widetilde{w_{n, i}}}^{2}(x)-\ln \left(1+\frac{{\widetilde{w_{n, i}}}^{2}(x)}{1+I\left(\varepsilon_{n} x+\varepsilon_{n} y_{n}^{i}\right)}\right)\right]+o_{\gamma}(1) \\
& \geq \sum_{i=1}^{k} \int_{\mathbb{R}^{2} \cap\left\{|x| \leq \frac{1}{\varepsilon_{n}}\right\}}\left|\nabla \widetilde{w_{n, i}}(x)\right|^{2}+\Gamma\left[\widetilde{w_{n, i}}{ }^{2}(x)-\ln \left(1+\frac{{\widetilde{w_{n, i}}}^{2}(x)}{1+I\left(\varepsilon_{n} x+\varepsilon_{n} y_{n}^{i}\right)}\right)\right]+o_{\gamma}(1) \\
& \geq \sum_{i=1}^{k} \int_{\mathbb{R}^{2} \cap\left\{|x| \leq \frac{1}{\varepsilon_{n}}\right\}}\left|\nabla \widetilde{w_{n, i}}(x)\right|^{2}+\Gamma\left[\widetilde{w_{n, i}}{ }^{2}(x)-\ln \left(1+\frac{{\widetilde{w_{n, i}}}^{2}(x)}{1+I_{2}-\delta}\right)\right]+o_{\gamma}(1) .
\end{aligned}
$$

By (2.30), (2.33), Lemma 2.4 and Lemma 2.9, and let $n \rightarrow+\infty$ and $\gamma \rightarrow 0$, one has

$$
\begin{aligned}
k m\left(\frac{1}{k}, I_{2}\right) & \geq \lim _{n \rightarrow+\infty} E_{\varepsilon_{n}}\left(u_{n}\right) \\
& \geq \sum_{i=1}^{k} \int_{\mathbb{R}^{2} \cap\left\{|x| \leq \frac{1}{\varepsilon_{n}}\right\}}\left|\nabla \widetilde{w_{n, i}}(x)\right|^{2}+\Gamma\left[{\widetilde{w_{n}, i}}^{2}(x)-\ln \left(1+\frac{\widetilde{w}_{n, i}{ }^{2}(x)}{1+I_{2}-\delta}\right)\right]+o_{\gamma}(1) \\
& \geq k \int_{\mathbb{R}^{2}}\left|\nabla w_{i}\right|^{2}+\Gamma\left[w_{i}^{2}-\ln \left(1+\frac{w_{i}^{2}}{1+I_{2}-\delta}\right)\right] \\
& >k \int_{\mathbb{R}^{2}}\left|\nabla w_{i}\right|^{2}+\Gamma\left[w_{i}^{2}-\ln \left(1+\frac{w_{i}^{2}}{1+I_{2}}\right)\right] \\
& \geq k m\left(\frac{1}{k}, I_{2}\right),
\end{aligned}
$$

a contradiction. Here we have used $w_{n, i} \rightarrow w_{i}$ in $H^{1}\left(B_{R}(0)\right)$, strongly in $L^{2}\left(B_{R}(0)\right)$ and and for each $\gamma>0$, there exists $R=R(\gamma)>0$, such that $\int_{B_{R}(0)} w_{i}^{2} \geq \frac{1}{k}-\gamma, i=1,2, \ldots, k$.

Therefore, there exists a subsequence $\varepsilon_{n}$ such that $\bar{x}_{n}^{i} \equiv \varepsilon_{n} y_{n}^{i} \rightarrow x_{0}^{i}, w_{n, i} \rightarrow w_{i} \geq 0$ in $H^{1}\left(\mathbb{R}^{2}\right)$ and a.e. in $\mathbb{R}^{2}$, where $x_{0}^{i}=g_{i} x_{0}, g_{i} \in G_{k}, i=1,2, \ldots, k$ and $g_{k} x_{0}=x_{0}$.

Applying the elliptic estimates theory to (2.31), we have $w_{n, i} \rightarrow w_{i}$ in $C_{l o c}^{2}\left(\mathbb{R}^{2}\right)$ and

$$
-\Delta w_{i}+\Gamma \frac{I\left(x_{0}^{i}\right)+w_{i}^{2}}{1+I\left(x_{0}^{i}\right)+w_{i}^{2}} w_{i}=\lambda_{0} w_{i}, x \in \mathbb{R}^{2}, i=1,2, \ldots, k,
$$

here $\lambda_{0}<0$ derives from Lemma 2.3 which implies $\lambda_{\varepsilon_{n}} \rightarrow \lambda_{0}$ as $\varepsilon_{n} \rightarrow 0^{+}$(up to a subsequence). 
Next, we will prove $I\left(x_{0}^{i}\right)=I_{2}, i=1,2, \ldots, k$.

Since $w_{n, i}(x)=u_{n}\left(x+y_{n}^{i}\right), i=1,2, \ldots, k$, and as $n \rightarrow+\infty, w_{n, i}(x) \rightarrow w_{i}(x)$ in $L^{2}\left(B_{R}(0)\right)$, by Lemma 3.1, Lemma 2.9, and the weakly lower semi-continuity of norm, we have

$$
\begin{aligned}
k m\left(\frac{1}{k}, I_{2}\right) & =\lim _{n \rightarrow+\infty} m_{\Gamma, k}\left(\varepsilon_{n}\right) \\
& =\lim _{n \rightarrow+\infty} \int_{\mathbb{R}^{2}}\left|\nabla u_{n}\right|^{2}+\Gamma\left[u_{n}^{2}-\ln \left(1+\frac{u_{n}^{2}}{1+I\left(\varepsilon_{n} x\right)}\right)\right] \\
& =\lim _{n \rightarrow+\infty} \sum_{i=1}^{k} \int_{B_{R}\left(y_{n}^{i}\right)}\left|\nabla u_{n}(x)\right|^{2}+\Gamma\left[u_{n}^{2}(x)-\ln \left(1+\frac{u_{n}^{2}(x)}{1+I\left(\varepsilon_{n} x\right)}\right)\right]+o_{\gamma}(1) \\
& =\lim _{n \rightarrow+\infty} \sum_{i=1}^{k} \int_{B_{R}(0)}\left|\nabla w_{n, i}\right|^{2}+\Gamma\left[w_{n, i}^{2}-\ln \left(1+\frac{w_{n, i}^{2}}{1+I\left(\varepsilon_{n} x+\varepsilon_{n} y_{n}^{i}\right)}\right)\right]+o_{\gamma}(1) \\
& =\lim _{n \rightarrow+\infty} \sum_{i=1}^{k} \int_{\mathbb{R}^{2}}\left|\nabla \widetilde{w_{n, i}}(x)\right|^{2}+\Gamma\left[\widetilde{w_{n, i}}(x)-\ln \left(1+\frac{w_{n, i}{ }^{2}(x)}{1+I\left(\varepsilon_{n} x+\varepsilon_{n} y_{n}^{i}\right)}\right)\right]+o_{\gamma}(1) \\
& =k \int_{\mathbb{R}^{2}}\left|\nabla w_{i}\right|^{2}+\Gamma\left[w_{i}^{2}-\ln \left(1+\frac{w_{i}^{2}}{1+I\left(x_{0}^{i}\right)}\right)\right]+o_{\gamma}(1) \\
& \geq k \int_{\mathbb{R}^{2}}\left|\nabla w_{i}\right|^{2}+\Gamma\left[w_{i}^{2}-\ln \left(1+\frac{w_{i}^{2}}{1+I_{2}}\right)\right]+o_{\gamma}(1) \\
& \geq k m\left(\frac{1}{k}, I_{2}\right)+o_{\gamma}(1) .
\end{aligned}
$$

Letting $\gamma \rightarrow 0$, this implies that $I_{2}=I\left(x_{0}^{i}\right), i=1,2, \ldots, k$. By (B2), we let $e_{1} \in\left\{x_{0}^{1}, x_{0}^{2}, \ldots, x_{0}^{k}\right\} \subset S^{1}$, then $\left|e_{1}\right|=1$.

At last, combining Lemma 3.2 and the proof process of Lemma 3.2, we can obtain

Lemma 3.3. For $\Gamma<\Gamma_{0}$ fixed, let $u_{\varepsilon}$ be a minimizer of $m_{\Gamma, k}(\varepsilon)$, then we have

$$
\left\|u_{\varepsilon}-\sum_{i=1}^{k} w_{i}\left(x+\frac{g_{i} e_{1}}{\varepsilon}\right)\right\|_{H^{1}\left(\mathbb{R}^{2}\right)} \rightarrow 0 \text {, as } \varepsilon \rightarrow 0 \text { (up to a subsequence), }
$$

where $w_{i}(x)$ is the minimizer of $m\left(\frac{1}{k}, I_{2}\right)$ and $e_{1} \in S^{1}=\left\{x \in \mathbb{R}^{2}|| x \mid=1\right\}$.

Acknowledgement: X.M. Wang is partially supported by NSFC-11861053 and the NSF of Jiangxi Province20192BAB201011. Z.-Q. Wang is partially supported by NSFC-11771324 and 11831009 . The authors thank the referee for helpful comments.

\section{References}

[1] N.K. Efremidis, S. Sears, D.N. Christodoulides, J.W. Fleischer, M. Segev, Discrete solitons in photorefractive optically induced photonic lattices, Phys. Rev. E. 66, (2002), 046602.

[2] N.K. Efremidis, J. Hudock, D.N. Christodoulides, J.W. Fleischer, O. Cohen, M. Segev, Two-dimensional optical lattice solitons, Phys. Rev. Lett. 91, (2003), no. 21, 213906.

[3] S. Gatz and J. Herrmann, Propagation of optical beams and the properties of two-dimensional spatial solitons in media with a local saturable nonlinear refractive index, J. Opt. Soc. Amer. B, 14, (1997), no. 7, 1795-1806.

[4] T.-C. Lin, M.R. Belić, M.S. Petrović, G. Chen, Ground states of nonlinear Schrödinger systems with saturable nonlinearity in $\mathbb{R}^{2}$ for two counterpropogating beams, Journal of Math. Physics, 55, (2014), 011505.

[5] T.-C. Lin, M.R. Belić, M.S. Petrović, N.B. Aleksić, G. Chen, Ground-state counterpropogating solitons in photorefractive media with saturable nonlinearity, J. Opt. Soc. Amer. B, 30, (2013), 1036-1040. 
[6] L.A. Maia, E. Montefusco, B. Pellacci, Weakly coupled nonlinear Schröodinger systems: the saturation effect, Calc. Var. Partial Differential Equations, 46, (2013), 325-351.

[7] L.A. Maia, E. Montefusco, B. Pellacci, Singularly perturbed elliptic problems with nonautonomous asymptotically linear nonlinearities, Nonlinear Analysis, 16, (2015), 193-209.

[8] L.A. Maia and R. Ruviaro, Positive and nodal solutions of nonlinear Schrd̈inger equations in a saturable medium, $A d v$. Nonlinear Stud., 15, (2015), 191-219.

[9] X.M. Wang, T.-C. Lin, Z.-Q. Wang, Existence and concentration of ground states for saturable nonlinear Schrödinger equations with intensity functions in $\mathbb{R}^{2}$, Nonlinear Analysis, 173, (2018), 19-36.

[10] Z.-Q. Wang, Construction of multi-peaked solutions for a nonlinear Neumann problem with critical exponent in symmetric domains, Nonlinear Analysis, 27, (1996), no. 11, 1281-1306.

[11] Z.-Q. Wang, Existence and symmetry of multi-bump solutions for nonlinear Schroödinger equations, Journal of Differential Equations, 159, (1999), 102-137.

[12] S. Maier, K. Schmitt, Z.-Q. Wang, On Neumann problems for semilinear elliptic equations with critical nonlinearity: existence and symmetry of multi-peaked solutions, Comm. Partial Differential Equations, 22, (1997), 1493-1527.

[13] F. Catrina and Z.-Q. Wang, Nonlinear elliptic equations on expanding symmetric domains, J. Differential Equations, 156, (1999), 153-181.

[14] T.-C. Lin, X.M. Wang, Z.-Q. Wang, Orbital stability and energy estimate of ground states of saturable nonlinear Schrödinger equations with intensity functions in $\mathbb{R}^{2}$, Journal of Differential Equations, 263, (2017), no. 8, 4750-4786.

[15] M. Willem, Minimax Theorems, Progress in Nonlinear Differential Equations and their Applications, 24, Birkhäuser Boston, Inc., Boston, MA, 1996.

[16] B. Simon, Schrödinger semigroups, Bull. Am. Math. Soc., 7, (1982), no. 3, 447-526.

[17] P.L. Lions, The concentration-compactness principle in the calculus of variations. The locally compact case. part 1, Ann. Inst. Henri Poincare, 1, (1984), no. 2, 109-145.

[18] P.L. Lions, The concentration-compactness principle in the calculus of variations. The locally compact case. part 2, Ann. Inst. Henri Poincare, 1, (1984), no. 4, 223-283.

[19] H. Brezis and E. Lieb, A relation between pointwise convergence of functions and convergence of functionals, Proc. Amer. Math. Soc., 88, (1983), no. 3, 486-490. 